\title{
Aperture-Coupled Asymmetric Dielectric Resonators Antenna for Wideband Applications
}

\author{
A.H. Majeed, A.S. Abdullah, F. Elmegri, K.H. Sayidmarie, R.A. Abd-Alhameed and J.M. Noras
}

\begin{abstract}
A compact dielectric resonator antenna (DRA) for wideband applications is proposed. Two cylindrical dielectric resonators which are asymmetrically located with respect to the center of a rectangular coupling aperture are fed through this aperture. By optimizing the design parameters, an impedance bandwidth of about $29 \%$, covering the frequency range from $9.62 \mathrm{GHz}$ to $12.9 \mathrm{GHz}$, and a gain of $8 \mathrm{~dB}_{\mathrm{i}}$ are obtained. Design details of the proposed antenna and the results of both simulation and experiment are presented and discussed.
\end{abstract}

Index Terms: Dielectric Resonator Antenna (DRA), aperture coupling, asymmetric shape.

\section{INTRODUCTION}

Since the first proposal in 1983 [1], dielectric resonator antennas (DRAs) have received increasing interest due to their many attractive features, such as high radiation efficiency, smaller size, the freedom to design their shape (rectangular, cylindrical, spherical, etc.) and their feeding structure, for example probe, microstrip line, slot or coplanar line. However, one major drawback of the DRA is limited bandwidth. For a single-mode excitation, the bandwidth is often below $10 \%$, which is not sufficient for many wideband applications. To overcome this limitation, various bandwidth enhancement techniques have been developed over the last few decades. One approach is to utilize different features of the dielectric resonators (DRs), such as structures of high aspect ratio [2], stacked multiple DRs with different materials to merge multi-resonance operation [3- 4], and inserting an air gap in the DR to lower the Q-factor [5-6]. In [7] a single cylindrical DR is excited by two crossed slots. The centers of the two slots are set at different positions and taking into consideration the partial independence of the slot modes from the DRA mode, a wider bandwidth was attained.

In this paper, a novel wideband slot-fed asymmetric dielectric resonator antenna is presented. A pair of cylindrical DRs is placed adjacently and asymmetrically with respect to the feeding rectangular aperture. A single slot here feeds the two DRs in such a way that more design freedom can be obtained, as the two DRAs can resonate at slightly different frequency resulting in a wider bandwidth.

\section{ANTENNA GEOMETRY}

The geometry of the proposed asymmetric wide band antenna is illustrated in Figure 1. The prototype antenna is fabricated on $30 \mathrm{~mm} \times 25 \mathrm{~mm}$ FR4 substrate with relative permittivity of $\varepsilon_{r s}=4.5$, a loss tangent of 0.017 , and a thickness $t=0.8 \mathrm{~mm}$. The feed microstrip line is placed symmetrically with respect to the coupling aperture. The microstrip line dimensions were calculated using empirical formulas given in [7] resulting in length $l_{f}=22.5 \mathrm{~mm}$ and width $w_{f}=1.5 \mathrm{~mm}$. A rectangular aperture (slot) of length $s_{l}$ and width of $s_{w}$ is etched on the ground plane. The dimensions of the aperture influence the resonant frequency of the structure and the amount of the undesired radiation in the back direction of the antenna. They also determine the coupling between the radiating DRs and the microstrip line. At the end of feed line there is a 'stub' of length $l_{\text {stub }}$ as shown in Figure 1. If the slot is modeled as a shunt impedance connected to the feed line, then the stub can be visualized as a reactance canceller. Practical experience has shown that the stub length should be close to $\lambda_{\mathrm{g}} / 4$ [8], and optimization started from this point. An alumina material of alumina-96pct, with $\varepsilon_{r d}=9.4$, diameter $D=6 \mathrm{~mm}$ and a height $h=9 \mathrm{~mm}$ is used for the DR structure. The two DRs are offset from the center of the slot $\left(y_{d} \neq y_{d l}\right)$ as shown in Figure1 (a).

The resonant frequency of a single segment cylindrical DRA (CDRA) excited in the $\mathrm{HEM}_{11 \delta}$ mode can be written as [9],

$$
f_{r}(G H z)=\frac{c}{2 \pi a \sqrt{\varepsilon_{r}}}\left(1.71+\frac{a}{h}+0.1578\left(\frac{a}{2 h}\right)^{2}\right)
$$

where $a=D / 2$, with $D$ the diameter of the CDRA, $c$ the velocity of light, $h$ the height of the CDRA above ground plane, and $\varepsilon_{\mathrm{r}}$ the relative permittivity of the CDR material. With dimensions $\mathrm{D}=6 \mathrm{~mm}, \mathrm{~h}=9 \mathrm{~mm}$ and $\varepsilon_{\mathrm{r}}=9.4$, the calculated resonance frequency according to Equation 1 was found to be $10.63 \mathrm{GHz}$. The slot length and the permittivities of the substrate and the DRA determine the frequency of the slot resonance. The DRA modes depend on the DR dimensions and permittivity, as well as on the feeding mechanism. Since the two DRAs are asymmetrically located with respect to the slot, then two nearby resonance frequencies are excited leading to wide bandwidth operation.

\section{PARAMETRIC STUDY}

In this section the influences of various parameters on the response of DRA antennas are discussed. The antenna structure is analyzed and optimized using Computer Simulation Technology (CST) microwave studio suite 2011, which is an electromagnetic simulator based on a finite integration technique (FIT) [10]. Figure 2 shows the simulated reflection coefficient as function of frequency for various slot lengths $s_{l}$. As the aperture length is reduced the 
input resistance of the antenna decreases. This might be thought of as decreasing the coupling factor between the feed line and the antenna. The slot length also affects the coupling to the DR, as can be seen from the fact that best matching is obtained for a slot length of $s_{l}=6.6 \mathrm{~mm}$. This analysis shows that slot length has most effect on the reflection coefficient and the resonant frequency, but it also affects the impedance bandwidth of the antenna to a lesser extent.

Figure 3 shows the simulated reflection coefficient of the DRA with slot width $s_{w}$ varied from $0.6 \mathrm{~mm}$ to $1.4 \mathrm{~mm}$. It is clear that the optimum impedance bandwidth is achieved at a slot width of $\mathrm{s}_{\mathrm{w}}=1.2 \mathrm{~mm}$.

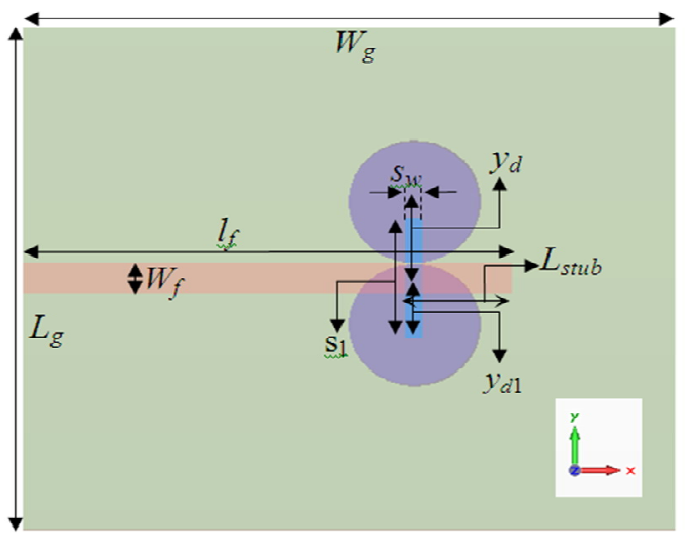

(a)

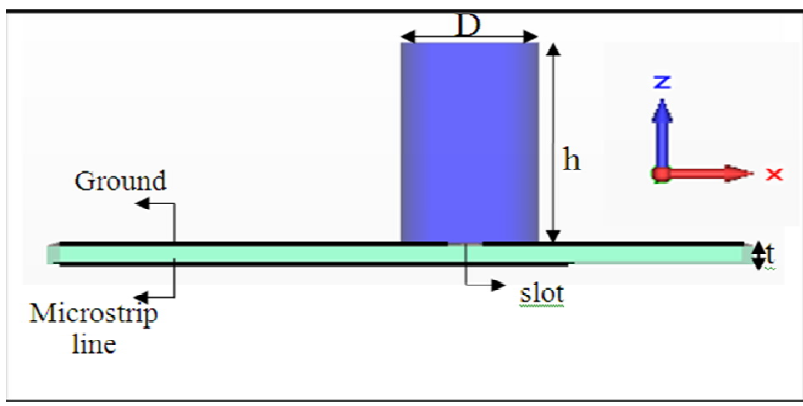

(b)

Fig. 1: Aperture-coupled asymmetric dielectric resonator antenna (a) top view and (b) side view with design dimensions and parameters

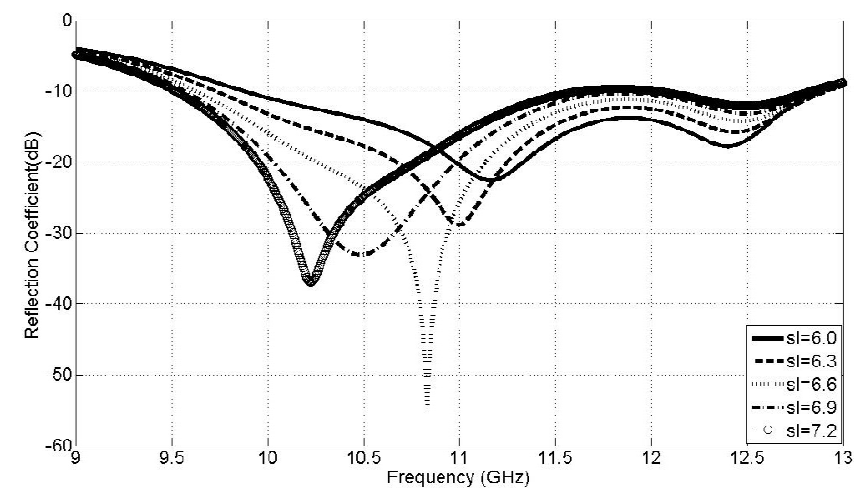

Fig. 2: Simulated reflection coefficient as a function of frequency for different slot lengths $s_{l}$ with $s_{w}=1.1 \mathrm{~mm}, l_{s t u b}=4 \mathrm{~mm}, y_{d}=3.75 \mathrm{~mm}, y_{d l}=-2.1 \mathrm{~mm}$.

The effect of the stub length $l_{\text {stub }}$ is shown in Figure 4, where it is clear that tuning the stub length can affect the bandwidth and matching of the resonant modes. The design is optimized at $l_{\text {stub }}=4 \mathrm{~mm}$, a value close to the estimated one of $\lambda_{\mathrm{g}} / 4=4.08 \mathrm{~mm}$.

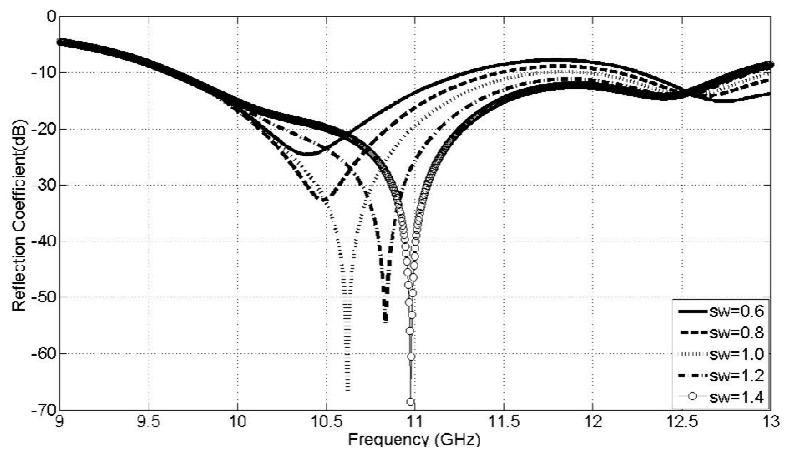

Fig. 3: Simulated reflection coefficient as a function of frequency for different slot widths $s_{w}$ with $s_{l}=6.6 \mathrm{~mm}, l_{s t u b}=4 \mathrm{~mm}, y_{d}=3.75 \mathrm{~mm}, y_{d 1}=-$ $2.1 \mathrm{~mm}$.

The effect of the stub length $l_{\text {stub }}$ is shown in Figure 4, where it is clear that tuning the stub length can affect the bandwidth and matching of the resonant modes. The design is optimized at $l_{\text {stub }}=4 \mathrm{~mm}$, a value close to the estimated one of $\lambda_{\mathrm{g}} / 4=4.08 \mathrm{~mm}$.

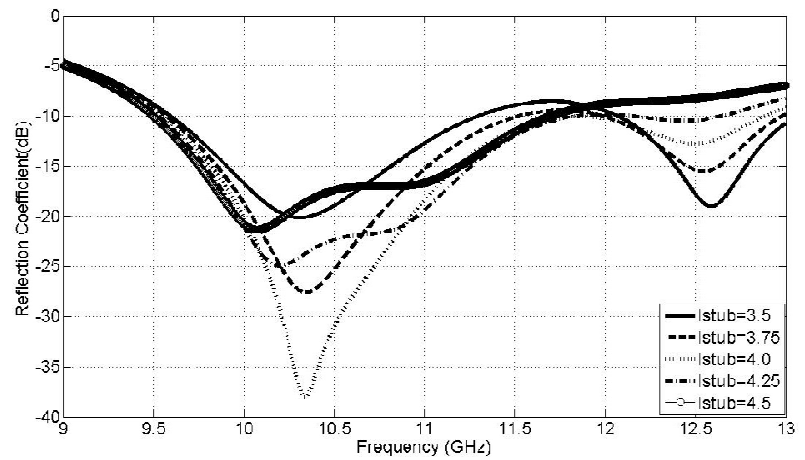

Fig. 4: Simulated reflection coefficient as a function of frequency for various stub lengths $l_{s t u b}$ with $s_{l}=6.6 \mathrm{~mm}, s_{w}=1.2 \mathrm{~mm}, y_{d}=3.75 \mathrm{~mm}, y_{d l}=-$ $2.1 \mathrm{~mm}$.

The effects of asymmetric location of the two DR's were investigated by moving one of the DRs along the length of the slot. The results obtained by moving the upper DR are shown in Figure 5, where it can be seen that the position of the DR affects the bandwidth and matching. A good compromise has been obtained for the position $y_{d}=3.75$ $\mathrm{mm}$. Figure 6 shows that moving the lower DRs along the length of the slot affects the bandwidth and matching, and a good compromise has been obtained for the position $y_{d l}=$ $2.3 \mathrm{~mm}$. Thus the off-set positioning has given one more degree of freedom for the design optimization. The two values $\left(y_{d}=3.75 \mathrm{~mm}\right.$ and $\left.y_{d l}=-2.3 \mathrm{~mm}\right)$ have been chosen 
as they give best response in bandwidth and matching as well as resulting in the same resonance frequency of 10.832 $\mathrm{GHz}$.

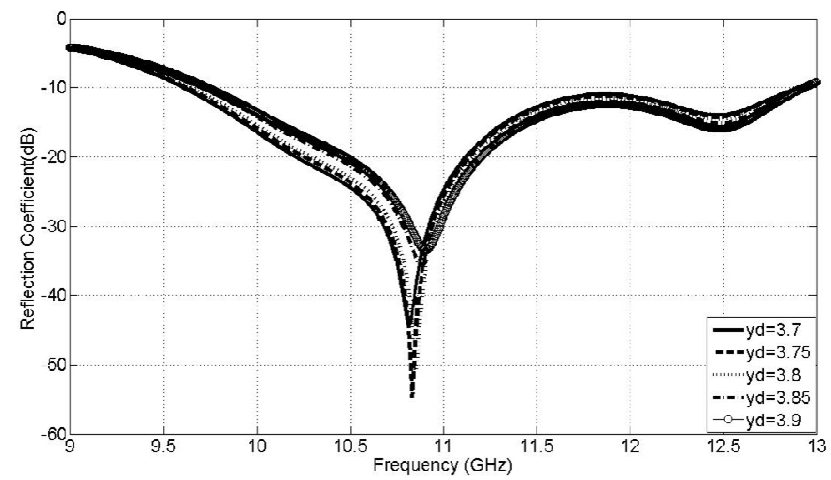

Fig. 5: Simulated reflection coefficient as a function of frequency for varying position of upper DR $y_{d}$ with $s_{l}=6.6 \mathrm{~mm}, s_{w}=1.2 \mathrm{~mm}, l_{l}=4 \mathrm{~mm}, y_{d l}=-$ $2.1 \mathrm{~mm}$.

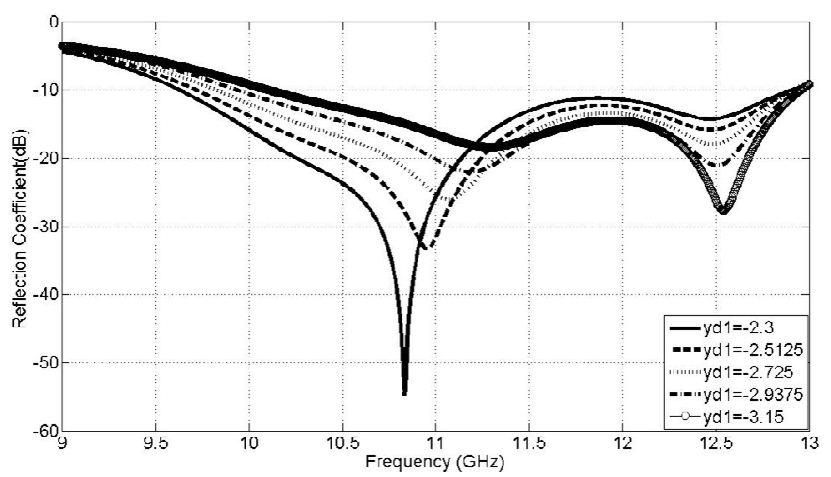

Fig. 6: Simulated reflection coefficient as a function of frequency for varying position of lower DR $y_{d l}$ with $s_{l}=6.6 \mathrm{~mm}, s_{w}=1.2 \mathrm{~mm}, l_{\text {stub }}=4 \mathrm{~mm}$, $y d=3.75 \mathrm{~mm}$.

\section{THE OPTIMIZED DESIGN}

Based on the detailed parametric studies, the optimum dimensions obtained for the antenna are listed in Table 1, and used in the fabrication of the antenna shown in Fig. 7. The antenna performance was measured with an HP8510C vector network analyzer. The measured and simulated reflection coefficients of the proposed antenna are shown in Fig. 8. The differences between the measured and simulated results, seen in a shift to higher frequency and general increase in $S_{11}$, may be attributed to the effects of fabrication inaccuracies, and of the use of glue to fix the DRA, as has been noticed previously [9]. The presented antenna here achieves an impedance matching $\left(\mathrm{S}_{11}<-10 \mathrm{~dB}\right.$ band) from $9.62 \mathrm{GHz}$ to $12.9 \mathrm{GHz}$, i.e. $29 \%$.

Table 1: Dimensions of the optimized antenna.

\begin{tabular}{|l|l|l|l|l|l|l|l|}
\hline parameter & $L_{g}$ & $W_{g}$ & $L_{f}$ & $W_{f}$ & $D$ & $h$ & $s_{l}$ \\
\hline $\begin{array}{l}\text { Optimum } \\
\text { value/mm }\end{array}$ & 30 & 25 & 21 & 1.5 & 6 & 9 & 6.6 \\
\cline { 1 - 5 } parameter & $s w$ & Lstub & $y d$ & $y d 1$ & & & \\
\cline { 1 - 4 } $\begin{array}{l}\text { Optimum } \\
\text { value/mm }\end{array}$ & 1.2 & 4 & 3.75 & -2.3 & & & \\
\hline
\end{tabular}
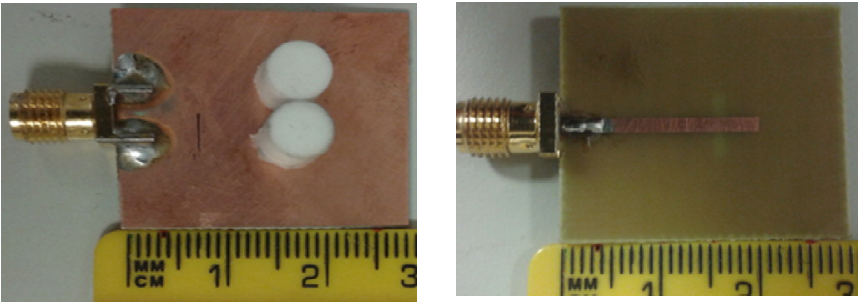

Fig. 7: Photograph of the fabricated antenna (a) front view and (b) back view

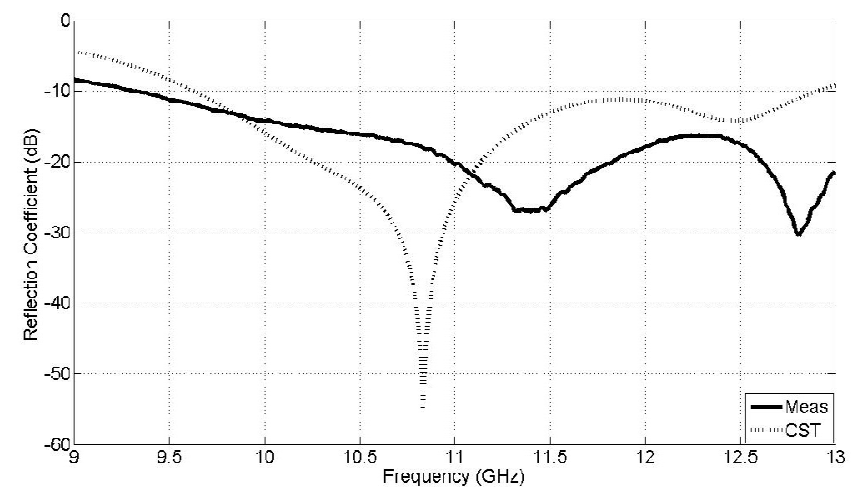

Fig. 8: Simulated and measured reflection coefficient of the proposed DRA

Figure 9 illustrates the simulated and measured antenna gain in the broadside direction of the proposed antenna. It should be noted that the simulated gain curve assumes an ideal feeding network, whereas the measured results include the insertion loss of the feeding network used, hence there are local discrepancies. The figure shows that the calculated gain varies between $5.47 \mathrm{~dB}_{\mathrm{i}}$ and $8 \mathrm{~dB}_{\mathrm{i}}$ with a maximum of $8 \mathrm{~dB}_{\mathrm{i}}$ at $12.8 \mathrm{GHz}$, while the measured gain varies between $6.34 \mathrm{dBi}$ and $7.72 \mathrm{dBi}$ across the pass band of 9.62-12.9 $\mathrm{GHz}$ and it can be said that on average the measurements are comparable with the prediction.

Figure 10 shows the simulated and measured impedance for the proposed antenna. The real parts are close to $50 \Omega$, while the imaginary parts fluctuate around zero. The slight differences between the simulated and measured impedances are due to the fabrication inaccuracies.

Measurements of the far-field radiation patterns of the prototype antenna were carried out in an anechoic chamber using an elevation-over-azimuth positioner, with the elevation axis coincident with the polar axis $\left(\theta=0^{\circ}\right)$ of the antenna's co-ordinate system. The azimuth drive thus generates cuts at constant $\phi$.

The fixed transmitting antenna was a broadband horn (EMCO type 3115 ) positioned $4 \mathrm{~m}$ from the antenna being tested. The azimuth positioner was rotated from $\theta=-180^{\circ}$ to $180^{\circ}$ at increments of $5^{\circ}$ for the selected measurement. Two pattern cuts, $\phi=0^{\circ}$ and $90^{\circ}$ were taken at two selected operating frequencies for which the matching was optimal. Figure 11 shows the simulated and measured normalized radiation patterns at resonance frequencies of $10.8 \mathrm{GHz}$ and $12 \mathrm{GHz}$. This shows the antenna has a wide radiation field covering half of space. The field has a low crosspolarization component and is mainly linearly polarized. 
The back lobe in the radiation pattern is due to a small amount of radiation from the slot.

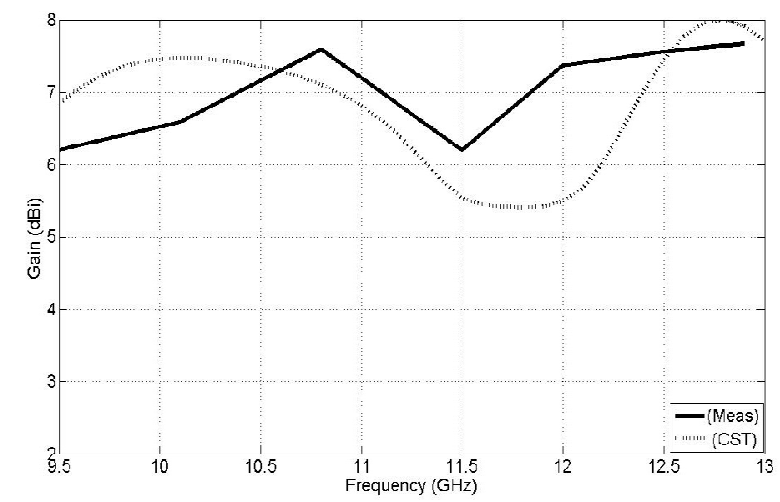

Fig. 9: Comparison of simulated and measured antenna gain of the proposed antenna

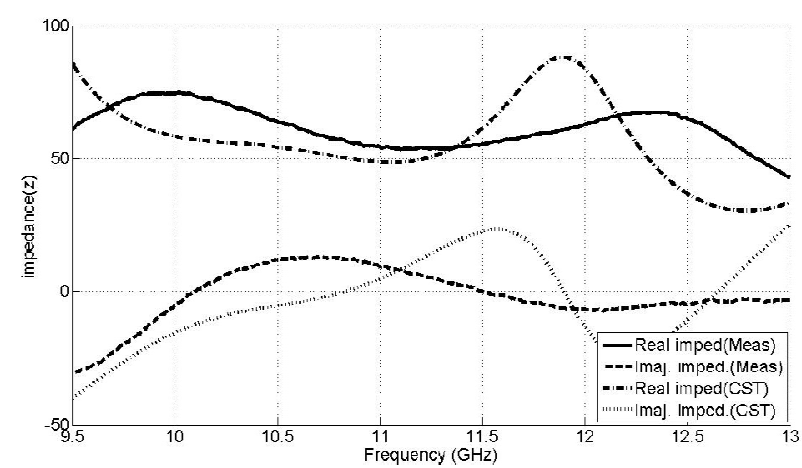

Fig. 10: Simulated and measured impedance of the proposed antenna

The magnitude of the electric and magnetic fields at 10.8 $\mathrm{GHz}$ and $12.3 \mathrm{GHz}$ are shown in Figure 12. The electric and magnetic fields distributions are plotted on the $\mathrm{xz}$ and $\mathrm{xy}$ planes respectively. It is observed that the magnetic field variations at $10.8 \mathrm{GHz}$ along specific azimuth direction at the base of each DRA, look quite similar and with intensity increasing at higher frequency. Hence, looking carefully at the variations of electric fields one can conclude that there is significant evidence of the appearance of a $\mathrm{TM}_{110} / \mathrm{HEM}_{11 \delta}$ mode of weakly uniform distribution at lower frequency $10.8 \mathrm{GHz}$ and a $\mathrm{TM}_{111} / \mathrm{HEM}_{112}$ mode of cycling field distribution at the higher frequency of $12.3 \mathrm{GHz}$. The asymmetry of such DRAs including the smallest ratio of radius-to-height ratio $(D / 2 h=1 / 3)$ supports the existence of close modes to work over a wide bandwidth [11].

\section{CONCLUSIONS}

A compact DRA for wideband applications has been demonstrated. The use of DRs can enhance the performance of the antenna. The asymmetric location of the pair of DRs can add another parameter for the designer to optimize the design. In this study, an impedance bandwidth of about $29 \%$, covering the frequency range from $9.62 \mathrm{GHz}$ to $12.9 \mathrm{GHz}$ and a realized gain of $8 \mathrm{~dB}_{\mathrm{i}}$ are obtained.
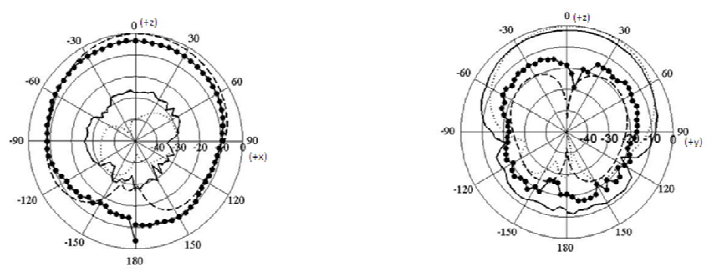

$10.8 \mathrm{GHz}$

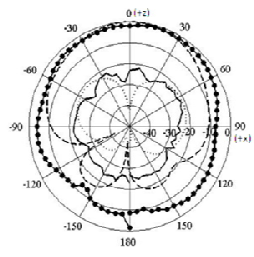

(a)

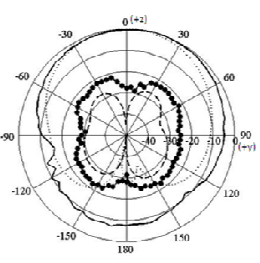

(b)
Fig. 11: Simulated and measured radiation pattern; (a) in xz plane, (b) in yz plane; simulated E $\theta$ : dashed line, simulated E $\phi$ : dotted line, measured

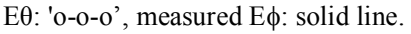
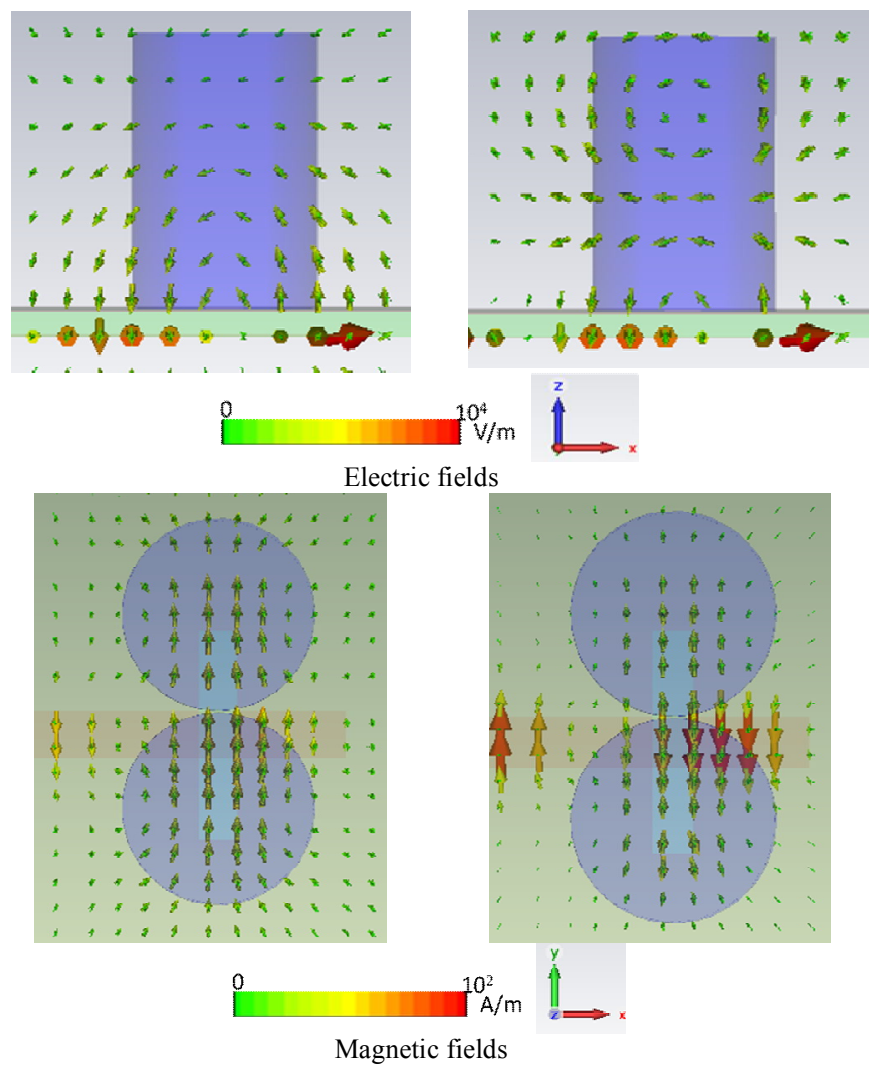

(a) $10.8 \mathrm{GHz}$

(b) $12.3 \mathrm{GHz}$

Fig. 12: Magnitude of electric (top, xz plane) and magnetic (bottom, xy plane) fields distribution for (a) $10.8 \mathrm{GHz}$, (b) $12.3 \mathrm{GHz}$.

\section{REFERENCES}

[1] S. A. Long, M. W. McAllister, and L. C. Shen, "The Resonant cylindrical dielectric cavity antenna," IEEE Trans. Antennas Propag., Vol.AP-31, No. 3, pp. 406-412, May 1983.

[2] A. Rashidian and D. M. Klymyshyn, "On the two segmented and high aspect ratio rectangular dielectric resonator antennas for bandwidth 
enhancement and miniaturization," IEEE Trans. Antennas Propag., Vol.57, No. 9, pp. 2775-2780, 2009.

[3] R. Chair, A. A. Kishk, K. F. Lee, and C. E. Smith, "Wideband flipped staired pyramid dielectric resonator antennas," Electron. Lett., Vol. 40, No. 10, pp. 581-582, 2004

[4] A. G. Walsh, S. D. Young, and S. A. Long, "An investigation of stacked and embedded cylindrical dielectric resonator antennas," IEEE Antennas Wireless Propag. Lett., Vol. 5, pp. 130-133, 2006.

[5] T. A. Denidni, Q. Rao, and A. R. Sebak, "Broadband L-shaped dielectric resonator antenna," IEEE Antennas Wirel. Propag. Lett., Vol. 4, pp. 453 $454,2005$.

[6] L. Z. Thamae and Z. Wu, "Broadband bowtie dielectric resonator antenna", IEEE Trans. Antennas Propag., Vol.58, No. 11, pp. 3707-3710, 2010.
[7] G. Almpanis, C. Fumeaux, and R. Vahldieck," Offset cross-slot-coupled dielectric resonator antenna for circular polarization", IEEE Microwave And Wireless Components Letters, VOL. 16, NO. 8, AUGUST 2006

[8] D. M. Pozar, "Microwave Engineering", 2nd edition, John Wiley \& Sons, New York, 1998

[9] A. A. Kishk, A. Ittipiboon, Y. M. M. Antar, and M. Cuhaci, "Slot excitation of the dielectric disk resonator," IEEE Trans. Antennas Propag.,Vol. 43, No. 2, pp. 198-201, Feb. 1995.

[10] CST: Microwave Studio based on the finite integration technique, 2011.

[11] Kwai-Man Luk, and Kwok-Wa Leung, Dielectric Resonator Antennas, by Research Studies Press ltd, Baldock, Hertfordshire, England, 2003. 